\title{
Summary of Working Group 4 : mixing and mixing-related $C P$ violation in the $B$ system
}

\author{
Alessandro Gaz* \\ Kobayashi-Maskawa Institute, Nagoya University, Nagoya, Japan \\ E-mail: gaz@hepl.phys.nagoya-u.ac.jp

\section{Vladimir V. Gligorov} \\ LPNHE, Université Pierre et Marie Curie, Université Paris Diderot, CNRS/IN2P3, Paris, France \\ E-mail: vgligorodlpnhe.in2p3.fr
}

\section{Dean Robinson}

University of Cincinnati, Cincinnati, Ohio, USA

E-mail: dean.robinsonauc.edu

\begin{abstract}
We summarize the new results on $B$ meson mixing and mixing-related $C P$ violation presented at CKM2016. We place these results in the context of both previous experimental measurements and the most recent theoretical developments in the field, and discuss prospects for ongoing and future mixing and mixing-related $C P$ violation measurements.
\end{abstract}

9th International Workshop on the CKM Unitarity Triangle

28 November - 3 December 2016

Tata Institute for Fundamental Research (TIFR), Mumbai, India

${ }^{*}$ Speaker. 


\section{Introduction}

Neutral beauty mesons may oscillate into their antiparticles, so that the physical states (those with well-defined masses and lifetimes) are admixtures of the flavor eigenstates. This mixing is parametrized by the magnitudes of the dispersive and absorptive components of the $\langle B|H| \bar{B}\rangle$ transition amplitude - a box loop in the standard model (SM) - as well as their relative phase, denoted $\phi_{d, s}$ in the $B^{0}$ and $B_{s}^{0}$ systems, respectively. The dispersive component generates mass splittings of the physical states, $\Delta m_{(d, s)}$, and is sensitive to heavy off-shell contributions from new physics (NP). The absorptive component generates width splittings $\Delta \Gamma_{(d, s)}$. It arises only from on-shell internal charm and up quark contributions, and is therefore less sensitive to possible NP effects.

Two types of mixing-related $C P$ violation arise in these systems. The first, $C P$ violation in mixing, occurs when the meson and anti-meson have different probabilities to oscillate into each other, and is predicted to be very close to zero in the SM with a very small theoretical uncertainty. The second, $C P$ violation in the interference of decay and mixing, occurs when both the meson and anti-meson can decay to the same final state, and the decay paths with and without intermediate mixing interfere. This kind of $C P$ violation is highly sensitive to the phase, $\phi_{d, s}$, and its absolute size depends on the final state in question.

Aside from their intrinsically fundamental nature, measurements of mass and width splittings and mixing-related $C P$ violation are of great interest because many of the observables can be predicted very precisely in the SM, and because new particles or force-carriers beyond the SM (BSM) can alter these predictions in experimentally observable ways. Many different experimental collaborations have contributed to our understanding of mixing in the $B$ system, from the initial discovery of $B$ mixing by the ARGUS collaboration [1], to precise measurements of $\Delta m_{(d, s)}$ and the CKM-angles $\alpha$ and $\beta$ at the $B$-factories and Tevatron, to recent precise measurements of $\phi_{s}, \Delta \Gamma_{s}$, and first precise mixing-related measurements of the CKM-angle $\gamma$ at ATLAS, CMS, and LHCb. At the same time, great progress has been made in making more precise theoretical predictions of the SM values of many of these quantities, making these experimental measurements sensitive probes of BSM physics.

The remainder of this document covers the current status of experimental measurements for each observable of interest, as well as their near-term outlook. Section 2 covers measurements of $\phi_{s}$ and $\Delta \Gamma_{s}$, section 3 measurements of $\Delta m_{(s, d)}$. Section 4 covers the measurements of photon polarization in radiative decays, while sections 5 to 7 cover measurements of the CKM angles $\alpha, \beta$, and $\gamma$, respectively. For historical reasons, measurements of $C P$ violation in neutral meson mixing are covered in the proceedings of WG2 [2]. We then discuss ongoing theoretical developments relevant to our WG in Sec. 8. Finally we conclude, and discuss the medium to long-term outlook for mixing-related measurements in the $B$ system.

\section{Measurements of $\phi_{s}$ and $\Delta \Gamma_{s}$}

The $\phi_{s}$ weak phase and $B_{s}^{0}$ meson decay width difference can be extracted by a time dependent full angular decay analysis of the $B_{s}^{0} \rightarrow J / \psi K^{+} K^{-}$decays. Such measurements have traditionally used the subset of decays where the $K^{+} K^{-}$pair originates in the decay of a $\phi$ meson, because 
its narrow width allows for an excellent background rejection, but since the conference the first measurement which uses the full $K^{+} K^{-}$mass spectrum [3] has become available, and more such measurements are expected in the future. The importance of $\phi_{s}$ is that, in the absence of significant loop-diagram contributions to $B_{s}^{0} \rightarrow J / \psi K^{+} K^{-}$decays ("penguin pollution"), its value is precisely predicted [4] in the SM

$$
\phi_{s}=-0.036 \pm 0.002
$$

and accurate experimental measurements of $\phi_{s}$ are therefore sensitive probes of the presence of BSM effects in the mixing and decay of $B_{s}^{0}$ mesons.

The results that the ATLAS [5] and CMS Experiments obtained on their Run1 datasets using $B_{s}^{0} \rightarrow J / \psi \phi$ decays are:

$$
\begin{aligned}
\phi_{s} & =-0.090 \pm 0.078(\text { stat }) \pm 0.041(\text { syst })[\mathrm{rad}] \\
\Delta \Gamma_{s} & =0.085 \pm 0.011(\text { stat }) \pm 0.007(\text { syst })\left[\mathrm{ps}^{-1}\right]
\end{aligned}
$$

for ATLAS [6] and:

$$
\begin{aligned}
\phi_{s} & =-0.075 \pm 0.097(\text { stat }) \pm 0.031(\text { syst })[\mathrm{rad}] \\
\Delta \Gamma_{s} & =0.095 \pm 0.013(\text { stat }) \pm 0.007(\text { syst })\left[\mathrm{ps}^{-1}\right]
\end{aligned}
$$

for CMS [7]. As can be seen in Fig. 1 both measurements are in excellent agreement with the SM expectations.
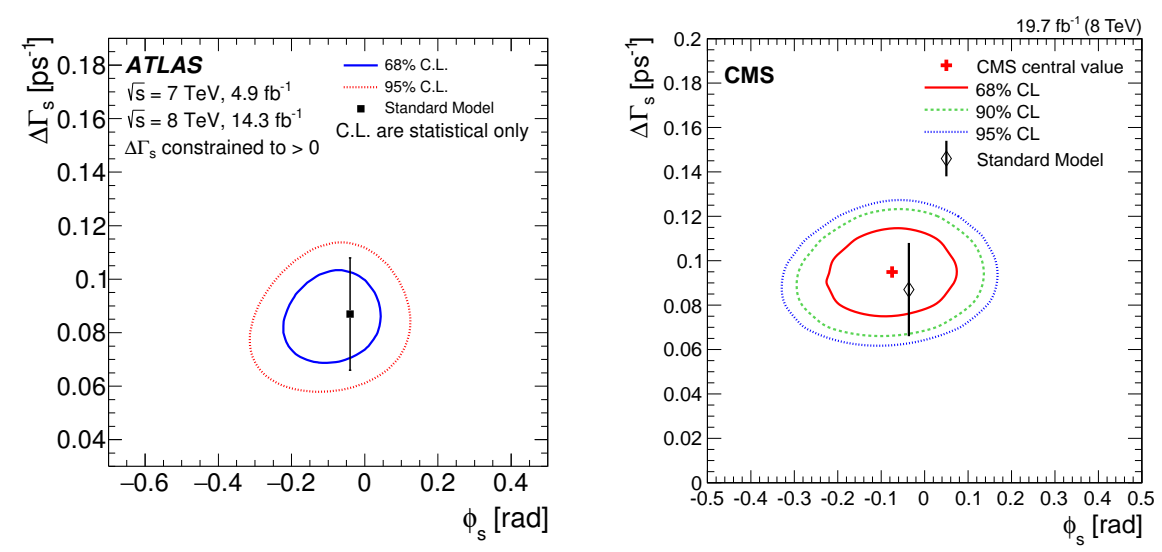

Figure 1: Results on $\phi_{s}$ and $\Delta \Gamma_{s}$ from $B_{s}^{0} \rightarrow J / \psi \phi$ decays from (left) ATLAS [6] and (right) CMS [7], reproduced from the respective citations.

The LHCb measurements of $\phi_{s}$ are covered in detail elsewhere in these proceedings [8]. LHCb uses not only $B_{s}^{0} \rightarrow J / \psi \phi$ but also $B_{s}^{0} \rightarrow J / \psi \pi^{+} \pi^{-}$decays, where the $\pi^{+} \pi^{-}$system is dominated by the $f_{0}(980)$ resonance and is greater than $97.7 \% C P$-odd. In this case no angular analysis is required, considerably simplifying the measurement and providing important complementarity of systematic uncertainties. The analysis of $B_{s}^{0} \rightarrow J / \psi \phi$ finds $\Delta \Gamma_{s}=0.0805 \pm 0.0091 \pm 0.0032 \mathrm{ps}^{-1}$, where the first uncertainty is statistical and the second systematic. A combination of $B_{s}^{0} \rightarrow J / \psi \phi$ and $B_{s}^{0} \rightarrow J / \psi \pi^{+} \pi^{-}$gives $\phi_{s}=-0.010 \pm 0.039 \mathrm{rad}$, which is the most precise single-experiment 
determination of this quantity. LHCb has also measured $\phi_{s}$ using $B_{s}^{0} \rightarrow \psi(2 S) \phi$ decays, obtaining $0.23_{-0.28}^{+0.29} \pm 0.02 \mathrm{rad}$.

While $B_{s}^{0} \rightarrow J / \psi K^{+} K^{-}$is expected to be dominated by the tree-level diagram, as the experimental precision on $\phi_{s}$ improves it will become increasingly important to account for residual penguin pollution [9] in order to correctly interpret any agreement, or otherwise, with the theoretical prediction. The size of such effects can be controlled using a combination of $B_{s}^{0} \rightarrow J / \psi K^{* 0}$ and $B^{0} \rightarrow J / \psi \rho$ decays, which are related by $\mathrm{U}$-spin symmetry and in the limit of zero non-factorizable $\mathrm{SU}(3)$ breaking are sensitive to the same, universal, penguin amplitudes and phases. LHCb has performed measurements of both modes $[10,11]$ and measures essentially zero penguin pollution to $\phi_{S}$ with a precision of around $15 \mathrm{mrad}$ in all three polarization-dependent phases. The LHCb measurement is statistics limited and penguin pollution to $\phi_{s}$ should therefore remain under control as the experimental precision approaches the SM value.

In addition to comparisons with the SM prediction, such tree-dominated measurements of $\phi_{s}$ can also be compared with the measurement of the related quantity $\phi_{S}^{s s \bar{s}}$ in the loop-dominated decays $B_{s}^{0} \rightarrow \phi \phi$ and $B_{s}^{0} \rightarrow K^{+} \pi^{-} K^{+} \pi^{-}$. In the absence of BSM physics effects, this quantity is expected to be very close to zero. LHCb has measured [12] $\phi_{s}^{s s \bar{s}}=-0.17 \pm 0.15 \pm 0.03 \mathrm{rad}$ using $B_{s}^{0} \rightarrow \phi \phi$, in excellent agreement with the SM prediction. The measurement, which uses $B_{s}^{0} \rightarrow K^{+} \pi^{-} K^{+} \pi^{-}$, is ongoing; it is considerably more challenging and requires a two-dimensional Dalitz analysis to account for the interfering intermediate resonances.

LHCb has also measured [13] the time-dependent $C P$ violation in $B^{0} \rightarrow \pi^{+} \pi^{-}$and $B_{s}^{0} \rightarrow K^{+} K^{-}$ decays. These can be interpreted ${ }^{1}$ in a combined U-spin analysis [15] as either measurements of $\gamma$ or $\phi_{s}$. Because the combined U-spin analysis is much less sensitive to U-spin breaking [16] when interpreted as a measurement of $\phi_{s}$, this is therefore the currently preferred way to interpret the measurements of these $C P$ observables.

$C P$ violation in $B^{0} \rightarrow \pi^{+} \pi^{-}$has been measured previously by BaBar [17] and Belle [18], while the measurement of $B_{s}^{0} \rightarrow K^{+} K^{-}$is unique to LHCb. Using a two-dimensional fit to the mass and decay-time of the neutral $B$ mesons, LHCb observes $C P$ violation in the interference of mixing and decay of $B_{s}^{0}$ mesons for the first time, and finds

$$
\begin{array}{ll}
C_{\pi \pi}=-0.24 \pm 0.07 \pm 0.01, & S_{\pi \pi}=-0.68 \pm 0.06 \pm 0.01, \\
C_{K K}=0.24 \pm 0.06 \pm 0.02, & S_{K K}=0.22 \pm 0.06 \pm 0.02, \\
A_{K K}^{\Delta}=-0.75 \pm 0.07 \pm 0.11, &
\end{array}
$$

where the first uncertainty is statistical and the second systematic. It can be seen that the (co)sinusoidal $C$ and $S$ parameters have negligible systematic uncertainties, while the hyperbolic $A_{K K}^{\Delta}$, which is sensitive to differences in the distributions of $B_{s}^{0}$ and $\overline{B_{s}^{0}}$ induced by $\Delta \Gamma_{s}$ at high decay-times, is much more sensitive to experimental effects. This is the most precise single-experiment determination of $S_{\pi \pi}$, and significantly improves the world-average of this parameter as shown in Fig. 2. A combined interpretation in terms of $\phi_{s}$ is not available at the time of writing but is expected to be performed in the future, and both $\mathrm{LHCb}$ and Belle-II are expected to improve our knowledge of

\footnotetext{
${ }^{1}$ In the absence of information about $B_{s}^{0} \rightarrow K^{+} K^{-}$, measurements of time-dependent $C P$ violation in $B^{0} \rightarrow \pi^{+} \pi^{-}$ have traditionally [14] been combined with other two-body $B^{0}$ decays in an isospin analysis to obtain a measurement of $\alpha$.
} 
the $C P$ observables in the future. The measurement of $A_{K K}^{\Delta}$ is a world first, allowing for a twofold reduction in the ambiguity of the $\phi_{S}$ determination and can also be interpreted as a measurement of $\Delta \Gamma_{s}$.

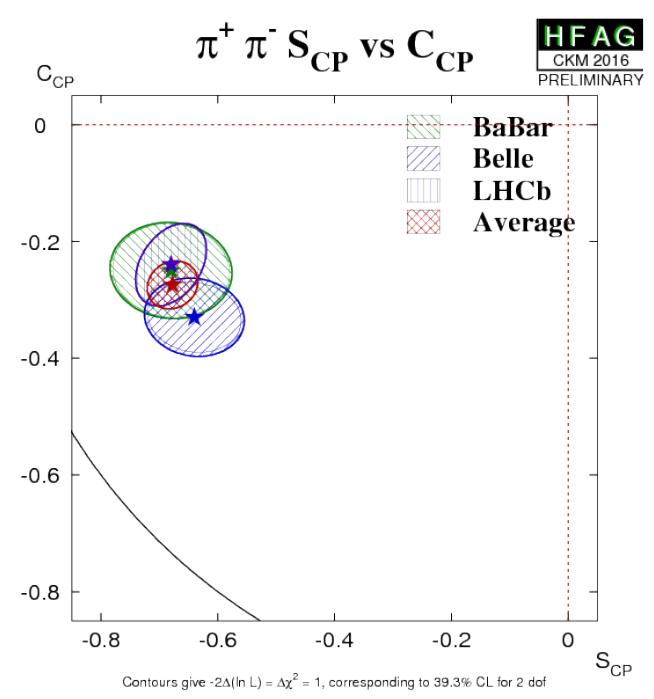

Figure 2: World average of time-dependent $C P$ observables in $B^{0} \rightarrow \pi^{+} \pi^{-}$, reproduced from HFAG [14].

\section{Measurements of $\Delta m_{(d, s)}$ and $\Delta \Gamma_{d}$}

The neutral $B$ meson mass splittings $\Delta m_{(d, s)}$ have been precisely measured by BaBar, Belle, $\mathrm{CDF}, \mathrm{D} 0$, and LHCb. The world average [14] of these measurements is currently dominated by the LHCb determinations, based on an analysis [19] of semileptonic $B^{0}$ decays in the case of $\Delta m_{d}$, and based on an analysis [20] of $B_{s}^{0} \rightarrow D_{s}^{-} \pi^{+}$decays in the case of $\Delta m_{s}$. LHCb finds

$$
\Delta m_{d}=(0.505 \pm 0.0021 \pm 0.0010) \mathrm{ps}^{-1}, \quad \Delta m_{s}=(17.768 \pm 0.023 \pm 0.006) \mathrm{ps}^{-1},
$$

where the first uncertainty is statistical, and the second systematic. Neither analysis is systematics limited, and LHCb is expected to update both measurements in the future. Belle-II will also be able to make a significant contribution to a precise measurement of $\Delta m_{d}$. A measurement of $\Delta m_{s}$ will be difficult for ATLAS and CMS because of a lack of efficient triggers for purely hadronic decays, but may become possible [21] in the HL-LHC era once their first-level tracking triggers come online, which would provide an important independent cross-check of LHCb's measurement.

The $B^{0}$ width splitting $\Delta \Gamma_{d}$ is predicted [22] to be $\Delta \Gamma_{d} / \Gamma_{d}=(4 \pm 1) \cdot 10^{-3}$ in the SM, and measuring it is therefore a useful null test of the SM [23]. Such tests are particularly important as a non-null value of $\Delta \Gamma_{d} / \Gamma_{d}$ could have important implications for the interpretation of $C P$ violation in the mixing of $B^{0}$ mesons, particularly in the context [24] of the D0 dimuon asymmetry measurement. LHCb has analyzed the effective decay-times of $B^{0} \rightarrow J / \psi K^{* 0}$ and $B^{0} \rightarrow J / \psi K_{\mathrm{S}}^{0}$ decays and obtains [25] $\Delta \Gamma_{d} / \Gamma_{d}=(-4.4 \pm 2.5 \pm 1.1) \cdot 10^{-2}$, while the current WA is dominated by the ATLAS measurement [26] of $\Delta \Gamma_{d} / \Gamma_{d}=(-0.1 \pm 1.1 \pm 0.9) \cdot 10^{-2}$, where the first uncertainties 
are statistical and the second systematic. While the systematic uncertainties are dominated by simulation sample sizes, both measurements are still an order of magnitude away from probing the SM prediction, so it will be important that even the subdominant systematics scale with luminosity if we hope to one day observe a non-null $\Delta \Gamma_{d} / \Gamma_{d}$ at its SM value.

The BaBar Collaboration studies the $B_{d}^{0}-{\overline{B_{d}}}^{0}$ oscillations to test the conservation of the $C P T$ symmetry [27]. At the lowest order in $|q / p|-1$ and $z$, the two mass eigenstates can be written:

$$
\begin{aligned}
B_{H} & =\left(p \sqrt{1+z} B^{0}-q \sqrt{1-z} \bar{B}^{0}\right) / \sqrt{2} \\
B_{L} & =\left(p \sqrt{1-z} B^{0}+q \sqrt{1+z} \bar{B}^{0}\right) / \sqrt{2}
\end{aligned}
$$

where:

$$
|q / p|=1-\frac{2 \mathfrak{I}\left(m_{12}^{*} \Gamma_{12}\right)}{4\left|m_{12}\right|^{2}+\left|\Gamma_{12}\right|^{2}}, \quad z=\frac{\left(m_{11}-m_{22}\right)-i\left(\Gamma_{11}-\Gamma_{22}\right) / 2}{\Delta m-i \Delta \Gamma / 2} .
$$

The test is performed by fitting the $C$ and $S$ parameters of the $C P$ violation in the interference between mixing and decay in $B \rightarrow c \bar{c} K_{S, L}^{0}$, when the other $B$ in the event decays to the $\ell^{ \pm} X$ final state, separating the cases when the decay of the $B$ to the $C P$-eigenstate happens before or after the decay of the other $B$ to the flavor-specific final state. The assumption $|\bar{A} / A|=1$, where $A(\bar{A})$ is the amplitude of $B^{0} \rightarrow c \bar{c} K^{0}\left(\bar{B}^{0} \rightarrow c \bar{c} \bar{K}^{0}\right)$ is not enforced.

The final result is:

$$
\begin{aligned}
\mathfrak{I}(z) & =0.010 \pm 0.030(\text { stat }) \pm 0.013(\text { syst }) \\
\mathfrak{R}(z) & =-0.065 \pm 0.028(\text { stat }) \pm 0.014(\text { syst }) \\
|\bar{A} / A| & =0.999 \pm 0.023(\text { stat }) \pm 0.017(\text { syst })
\end{aligned}
$$

in good agreement with $C P T$ conservation.

\section{Measurements of photon polarization in radiative decays}

Radiative decays such as $B_{s}^{0} \rightarrow \phi \gamma$ give access to information about the photon polarization, and tests the presence of possible right-handed couplings and set constraints on the Wilson coefficients $C_{7}$ and $C_{7}^{\prime}$ in EFT frameworks. Previous measurements [14] of photon polarization in radiative $B^{0}$ decays have been compatible with SM predictions.

$\mathrm{LHCb}$ is uniquely able to probe photon polarization in radiative $B_{s}^{0}$ decays, which offer a complementary test of BSM physics. While a full analysis requires tagging the production flavor of the $B_{s}^{0}$ meson, the large value of $\Delta \Gamma_{s}$ allows a measurement of the effective lifetime of $B_{s}^{0} \rightarrow \phi \gamma$ to be interpreted in terms of the $C P$-violating observable $A^{\Delta}$. LHCb performs this measurement [28] by normalizing the $B_{s}^{0} \rightarrow \phi \gamma$ decay-time distribution to that of $B^{0} \rightarrow K^{* 0} \gamma$, cancelling most experimental biases on the decay-time. The fitted mass and decay-time distributions are shown in Fig. 3; LHCb finds $A^{\Delta}=-0.98_{-0.52}^{+0.46}$, in $2 \sigma$ agreement with the SM prediction [29] of $A^{\Delta}=0.047 \pm 0.025 \pm 0.015$. The measurement is statistics limited, and future tagged analyses will enable the (co)sinusoidal $C P$ observables to be measured, allowing both the real and imaginary components of $C_{7}$ and $C_{7}^{\prime}$ to be constrained. 

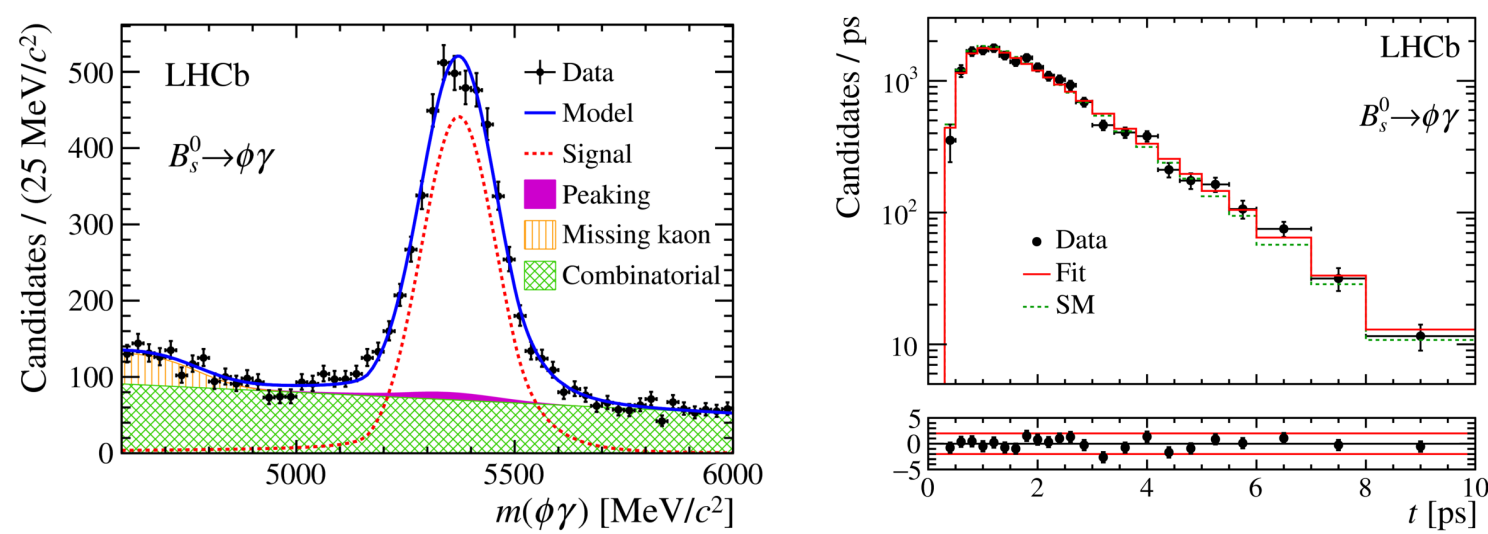

Figure 3: Distributions of the fitted $B_{s}^{0} \rightarrow \phi \gamma$ (left) invariant mass and (right) decay-time, reproduced from [28].

\section{Measurements of the CKM angle $\alpha$}

One of the possible ways to extract the CKM angle $\alpha$ is through an isospin analysis of the $B \rightarrow \rho \rho$ decays. The Belle Collaboration recently updated their measurements of the branching fraction, longitudinal polarization fraction, and $C P$-violation parameters of the $B^{0} \rightarrow \rho^{+} \rho^{-}$decay, using their full dataset [30]. The results are:

$$
\begin{aligned}
\mathscr{B}\left(B^{0} \rightarrow \rho^{+} \rho^{-}\right) & =[28.3 \pm 1.5(\text { stat }) \pm 1.5(\text { syst })] \times 10^{-6} \\
f_{L} & =0.988 \pm 0.012(\text { stat }) \pm 0.023(\text { syst }) \\
S & =-0.13 \pm 0.15(\text { stat }) \pm 0.05(\text { syst }) \\
C & =0.00 \pm 0.10(\text { stat }) \pm 0.06(\text { syst })
\end{aligned}
$$

Combining the results summarized above with previous $B \rightarrow \rho \rho$ measurements in an isospin analysis, one of the solutions is:

$$
\alpha=(93.7 \pm 10.6)^{\circ},
$$

in very good agreement with the SM.

\section{Measurements of the CKM angle $\beta$}

While the world average [14] of $\beta$ is still dominated by the BaBar [31] and Belle [32] measurements, LHCb also contributes [33] and can be expected to reach the BaBar and Belle individual sensitivities once Run II data and improvements in flavor tagging are included in the analysis. $\mathrm{LHCb}$ has also measured [34] $C P$ violation in $B_{s}^{0} \rightarrow J / \psi K_{S}^{0}$ decays, which can help to constrain the size of penguin contributions to the measurement of $\beta$ from $B^{0} \rightarrow J / \psi K_{S}^{0}$, as well as the $C P$ violating parameters in $B^{0} \rightarrow D^{+} D^{-}$decays, which can also be interpreted in terms of constraints on $\beta$. The current world average of measurements of $B^{0} \rightarrow D^{+} D^{-}$is shown in Fig. 4; interestingly while all measurements are compatible with each other, the LHCb measurement does not confirm the maximal (within uncertainty) Belle measurement of $S$. 


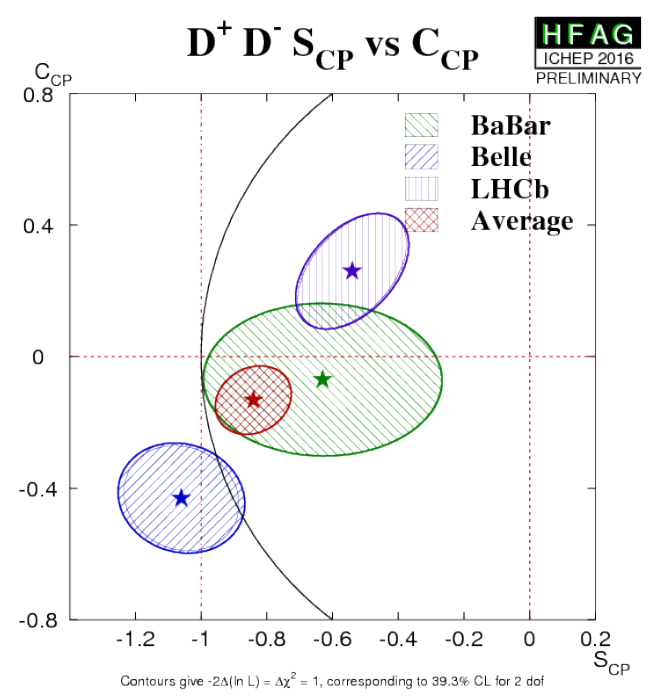

Figure 4: World average of time-dependent $C P$ observables in $B^{0} \rightarrow D^{+} D^{-}$, reproduced from HFAG [14].

The BaBar and Belle Collaboration obtained the first observation of $C P$-violation in the channels $B^{0} \rightarrow D_{C P}^{(*)} h^{0}$, where $h^{0}$ is a light unflavored neutral meson, by combining their final datasets [35]. Neglecting the very small contribution from the CKM suppressed amplitude $b \rightarrow u \bar{c} d$, the time dependent analysis is sensitive to $\sin (2 \beta)$. A joint likelihood, sharing the same physics parameters but independent background modeling parameters, the two experiments find:

$$
\begin{aligned}
-\eta_{f} S & =+0.66 \pm 0.10(\text { stat }) \pm 0.16(\text { syst }), \\
C & =+0.02 \pm 0.07(\text { stat }) \pm 0.03(\text { syst }),
\end{aligned}
$$

with the significance of the observation being 5.4 standard deviations.

\section{Measurements of the CKM angle $\gamma$}

Measurements of the CKM angle $\gamma$ require the interference of $b \rightarrow u$ and $b \rightarrow c$ transitions. Such interference can occur in the decays of charged as well as neutral $B$ hadrons, in tree-dominated transitions as well as transitions where both tree and loop diagrams contribute. The measurement of $\gamma$ from the tree-dominated decays of $B^{ \pm}$mesons, covered in the proceedings of WG5 [36], has a particular importance as it allows a purely tree-level ${ }^{2}$ determination of the apex of the unitarity triangle, and therefore a test of the self-consistency of the CKM mechanism of $C P$ violation when compared with determinations of $\alpha$ and $\beta$ in transitions where both tree and loop diagrams contribute. It is also, however, possible to measure $\gamma$ by exploiting $C P$ violation in the interference of mixing and decay of neutral $B$ mesons. These time-dependent determinations of $\gamma$ are particularly powerful in the case of $B_{s}^{0}$ mesons, because the large width difference between the light and heavy $B_{s}^{0}$ eigenstates

\footnotetext{
${ }^{2}$ Higher order box corrections [37] only enter at $\delta \gamma / \gamma \approx 10^{-7}$, well beyond any current or future experimental sensitivity.
} 
makes additional $C P$ observables available compared to the $B^{0}$ system and allows for a determination of $\gamma$ with fewer ambiguities.

A particularly powerful time-dependent measurement of $\gamma$, described in detail in these proceedings [38], utilizes the decay $B_{s}^{0} \rightarrow D_{s}^{ \pm} K^{\mp}$. In this case the $b \rightarrow u$ and $b \rightarrow c$ transitions are both of order $\lambda^{3}$, leading to large interference, and the large value of $\Delta \Gamma_{s}$ allows for a determination of $\gamma$ with only a twofold ambiguity. The preliminary result obtained with the full Run I LHCb dataset, which shows $3.6 \sigma$ evidence for $C P$-violation in this mode, is

$$
\begin{array}{lll}
\gamma=\left(127_{-22}^{+17}\right)^{\circ}, & \delta=\left(358_{-16}^{+15}\right)^{\circ}, & r_{D_{s}^{+} K}=0.37_{-0.09}^{+0.10},
\end{array}
$$

where $\delta$ is the $C P$-conserving angle between the $b \rightarrow u$ and $b \rightarrow c$ transitions, $r_{D_{s}^{+} K}$ is the amplitude ratio of the interfering diagrams, and the intervals for the angles are expressed modulo $180^{\circ}$. The uncertainties are a combination of statistical and systematic ones; the statistical uncertainties dominate and all systematic uncertainties are expected to scale with luminosity for the foreseeable future.

While not the most sensitive single-mode determination of $\gamma, B_{s}^{0} \rightarrow D_{s}^{ \pm} K^{\mp}$ plays a similar role in the overall LHCb combination [39] of $\gamma$ to that of the GGSZ measurement. Because of their twofold ambiguity, these measurements select the "correct" solution among the ones allowed by the most precise ADS/GLW measurement [40]. For this reason the determination of $\gamma$ from $B_{s}^{0} \rightarrow D_{s}^{ \pm} K^{\mp}$, which is only possible at $\mathrm{LHCb}$, will remain a key measurement for both the current and upgraded $\mathrm{LHCb}$ detectors. $\mathrm{LHCb}$ is also pursuing a measurement of time-dependent $C P$-violation in the decay mode $B^{0} \rightarrow D^{ \pm} \pi^{\mp}$, described in these proceedings [41], but no results are available yet. This measurement, previously performed by BaBar [42], [43] and Belle [44], [45] is much less sensitive than $B_{s}^{0} \rightarrow D_{s}^{ \pm} K^{\mp}$, both because of smaller interference and because the small value of $\Delta \Gamma_{d}$ leads to fewer accessible $C P$-observables. The much smaller $C P$ asymmetry also makes this measurement particularly sensitive the asymmetries in the flavor tagging of $B^{0}$ and $\bar{B}^{0}$ mesons. Nevertheless, it is expected that both LHCb and Belle-II will carry out this measurement in the future.

\section{Theory Developments}

\subsection{Lattice Calculations}

The most precise computation to date for $\left|V_{t d}\right|$ and $\left|V_{t s}\right|$, or alternatively $\Delta m_{d, s}$, is recently available from the Fermilab/MILC collaboration [46], building on previous results for $B$ mixing matrix elements $[47,48,49,50,51,52]$. These predictions are achieved via a three flavor lattice QCD calculation for the neutral $B$ mixing hadronic matrix elements, $\left\langle\bar{B}\left|\mathscr{O}_{i}^{q}\right| B\right\rangle$, with $\mathscr{O}_{i}^{q}$ combinations of scalar, pseudoscalar, vector and axial vector four-quark operators as required by the theory of interest. In the SM, the oscillation frequency

$$
\Delta m_{q}=\frac{G_{F}^{2} m_{W}^{2} M_{B_{q}}}{6 \pi^{2}} S_{0}\left(m_{t}^{2} / m_{W}^{2}\right) \eta_{2 B}\left|V_{t b} V_{t q}^{*}\right| f_{B_{q}}^{2} \hat{B}_{B_{q}}^{(1)}
$$

in which $\hat{B}_{B_{q}}^{(1)}$ is a renormalization-improved bag parameter associated with the operator product of left-handed quark currents $\mathscr{O}_{1}^{q}=\bar{b}^{\alpha} \gamma^{\mu}\left(1-\gamma^{5}\right) q^{\alpha} \bar{b}^{\beta} \gamma^{\mu}\left(1-\gamma^{5}\right) q^{\beta}$, while $S_{0}\left(x_{t}\right)$ and $\eta_{2 B}$ encode 
known electroweak and perturbative QCD corrections, respectively. Computation of $f_{B_{q}} \sqrt{\hat{B}_{B_{q}}^{(1)}}$ may be combined with either direct measurements of $\Delta m_{s, d}$ or CKM global fits to test the self-consistency of data with lattice calculations. One may also extract $\left|V_{t d} / V_{t s}\right|$ or $\Delta m_{d} / \Delta m_{s}$ via the flavor $S U$ (3) breaking ratio

$$
\xi=\sqrt{f_{B_{s}}^{2} \hat{B}_{B_{s}}^{(1)} / f_{B_{d}}^{2} \hat{B}_{B_{d}}^{(1)}}
$$

in which many theory uncertainties cancel. Computation of the other $B$ mixing matrix elements, corresponding to the other operators $\mathscr{O}_{i}^{q}$, permit similar predictions for BSM theories.

This lattice calculation is performed for $N_{f}=2+1$ light quark flavors. Non-perturbative renormalization effects are included, while two-loop chiral-continuum extrapolation of the lattice results determines the physical limits. Presentation of all crucial details may be found in Ref. [46]. Present results are

$$
\begin{gathered}
f_{B_{d}} \sqrt{\hat{B}_{B_{d}}^{(1)}}=227.7(9.5)(2.3) \mathrm{MeV}, \quad f_{B_{s}} \sqrt{\hat{B}_{B_{s}}^{(1)}}=274.6(8.4)(2.7) \mathrm{MeV}, \\
\xi=1.206(18)(6),
\end{gathered}
$$

currently the most precise predictions to date. Combination of these results with $\left|V_{t q}\right|$ results from CKM global fits yields

$$
\begin{gathered}
\Delta m_{d}^{\mathrm{CKM}}=0.630(53)(42)(5)(13) \mathrm{ps}^{-1}, \quad \Delta m_{s}^{\mathrm{CKM}}=19.6(1.2)(1.0)(0.2)(0.4) \mathrm{ps}^{-1}, \\
\Delta m_{d}^{\mathrm{CKM}} / \Delta m_{s}^{\mathrm{CKM}}=0.0321(10)(15)(0)(3),
\end{gathered}
$$

in an approximately $2 \sigma$ tension with direct measurements of these parameters [14]

$$
\Delta m_{d}^{\mathrm{HFAG}}=0.5064(19) \mathrm{ps}^{-1}, \quad \Delta m_{s}^{\mathrm{HFAG}}=17.757(21) \mathrm{ps}^{-1} .
$$

Alternatively, using the direct measurements for $\Delta m_{d, s}$ produces CKM predictions

$$
\begin{gathered}
\left|V_{t d}\right|=8.00(33)(2)(3)(8) \times 10^{-3}, \quad\left|V_{t s}\right|=39.0(1.2)(0.0)(0.2)(0.4) \times 10^{-3}, \\
\left|V_{t d} / V_{t s}\right|=0.2052(31)(4)(0)(10),
\end{gathered}
$$

approximately $\sim 2 \sigma$ below the results from global CKM fits [53, 54]. In particular, in the context of the $b \rightarrow d$ unitarity triangle, this tension manifests as a tension between the allowed regions for the $\mathrm{CP}$ violating parameter $\varepsilon_{K}$ and $\Delta m_{d} / \Delta m_{s}$, with potentially interesting theory implications.

These same lattice calculations also generate predictions for $B_{d, s} \rightarrow \mu \mu$ and $\Delta \Gamma_{d, s}$, with some mild tensions for $B_{d}$ decays. Further improved calculations for $N_{f}=2+1+1$ that include the charm quark sea effects and physical quark masses are anticipated.

\subsection{Constrained MFV theories}

Given the possible tensions in the unitarity triangle between $\varepsilon_{K}$ and $\Delta m_{d, s}$, it is informative to consider which classes of BSM theories could account for such tension. A class involving near-minimal BSM contributions are models of constrained minimal flavor violation (CMFV) $[55,56,57,58,59]$. In these theories, the SM Yukawa couplings $Y_{u}$ and $Y_{d}$ are treated as $U(3) \times U(3)$ flavor violating spurions, generating the sole source of $C P$ violation. BSM effects are encoded in higher dimensional SM effective operators. 
This class of theories preserves the unitarity structure of the CKM matrix. The precisely measured CKM matrix elements for the first two generations imply the relation, at percent level precision,

$$
R_{t} \equiv\left|\frac{V_{t b}^{*} V_{t d}}{V_{c b}^{*} V_{c d}}\right| \simeq \frac{\left|V_{t d} / V_{t s}\right|}{\lambda}
$$

where $\lambda$ is the usual Wolfenstein parameter. The ratio $\left|V_{t d} / V_{t s}\right|$ is determined precisely via Eq. (8.1) from direct measurements of $\Delta m_{d} / \Delta m_{s}$ and lattice computations of $\xi$. Thus, measurements of $\Delta m_{d, s}$ and the time-dependent $C P V$ observable $S_{\psi K_{S}}$, which determines $\sin (2 \beta)$ (see below), fully determine a 'universal unitarity triangle' (UUT) for the $b \rightarrow d$ system for all CMFV theories.

The electroweak loop function $S_{0}\left(x_{t}\right)$ in $\Delta m_{d, s}$ (8.1) also appears in the CP violating parameter $\varepsilon_{K}$. CMFV replaces $S_{0}\left(x_{t}\right)$ with a generalized universal function $S(v)$, bounded below by $S_{0}\left(x_{t}\right)$ in most compelling BSM scenarios. The UUT is, however, independent of the electroweak loop function, as it drops out of the $\Delta m_{d} / \Delta m_{s}$ ratio. Consequently, in CMFV theories, there is an extra degree of freedom between the predictions for $\left|V_{u b} / V_{c b}\right|$ and the CKM angle $\gamma$, both fixed by the UUT, and the constraints from measurements of $\varepsilon_{K}$.

This extra freedom is, however, found to be insufficient to relax the tension between $\Delta m_{d, s}$ and $\varepsilon_{K}$ generated in the FNAL/MILC lattice results, when applied together with the bound $S(v) \geq S_{0}\left(x_{t}\right)$ $[59,60]$. One may take either $\Delta m_{s, d}$ or $\varepsilon_{K}$ direct measurements as inputs, and thereby determine all other CKM matrix elements as functions of $S(v)$ via the UUT constraints: $\Delta m_{d, s}$ direct measurements imply an upper bound on $\varepsilon_{K}$ that is too small compared to data; $\varepsilon_{K}$ data implies lower bounds on $\Delta m_{d, s}$, that are above current measurements. If the tension between lattice and experimental data persists in the unitarity triangle, it will become imperative to consider new sources of flavor violation in $\Delta F=2$ processes, beyond CMFV models.

\subsection{Precision control of Penguin Pollution}

The time-dependent $C P V$ observable $S_{\psi K_{S}}$, generated by the interference of $B$-mixing and $B_{d} \rightarrow J / \psi K_{S}$ decay amplitudes, has long been considered a golden mode for extraction of $\phi_{d}$, via the relation $S_{\psi K_{S}}=\sin \left(\phi_{d}+\delta \phi_{d}\right)$. The latter 'penguin pollution' phase is expected to be CKM and loop-suppressed, yielding a clean measurement of $\phi_{d} \simeq 2 \beta$ in the SM. Similar techniques may be used to extract $\phi_{s}$ from, e.g., $B_{s} \rightarrow J / \psi \phi$. Estimates place $\delta \phi_{d} \lesssim 1^{\circ}[61,62,63,64,65,66,67,68$, 69], which is near to or larger than the expected precision of upcoming measurements, requiring theoretical control of these terms.

In general terms, two different paths have been followed to control these penguin pollution terms. Direct calculational approaches have been attempted using QCD factorization [61], or factorization combined with perturbative QCD [63], that yield very small estimates $\delta \phi_{d} \lesssim 0.1^{\circ}$. A more recent study [68] makes use of an OPE-type approach to directly calculate the penguin pollution contributions. This analysis integrates out the $u$-quark loop, associated with this penguin pollution term, on the basis that the typical momentum flow is large. One obtains a factorization formula for the penguin contributions, and one may show that soft and collinear divergences formally cancel or factorize up to $\Lambda_{\mathrm{qcd}} / m_{J / \psi}$ corrections. Large- $N_{c}$ arguments permit estimation of remaining uncertainties, yielding $\delta \phi_{d}<0.68^{\circ}$ and $\delta \phi_{s} \lesssim 1^{\circ}$.

A second, complementary path makes use of light quark flavor symmetries to constrain or eliminate penguin pollution effects in particular observables. For example on may make use of 
$U$-spin symmetry to control pollution of $S_{\psi K_{S}}$ with $B \rightarrow J / \psi \pi$ data, up to symmetry breaking effects $[62,64,65,67]$. Full $S U(3)$ analyses $[66,67,69]$ that treat all flavor symmetry breaking effects, or subsets of them, can be used to control penguin pollution. A simultaneous fit [66] predicts $\delta \phi_{d} \lesssim 0.6^{\circ}$. Flavor symmetry relations beyond the flavor symmetry limit [69] permit extraction of $\phi_{d}$ with penguin pollution suppressed by $S U(3)$ breaking, but require precision measurement of $C P$-averaged rates in the charged and neutral $B \rightarrow J / \psi K$ and $B \rightarrow J / \psi \pi$ systems.

\subsection{Lessons of isospin violation}

Measurement of CP averaged rates at the percent-level precision required for the use of abovementioned flavor $S U(3)$ results $[66,69]$ in turn requires careful inclusion of isospin violating effects in the ratio of neutral and charged $B$ production at the $\Upsilon(4 S)$ [70]. Knowledge of this ratio, $r_{+0}=$ $f_{+-} / f_{00}$, is crucial for precision measurement of branching ratios: the measured charged (neutral) $B$ branching ratios are known up to a $1+r_{+0}\left(1+1 / r_{+0}\right)$ factor, expected to be of the order of a few percent from unity. Control of this ratio can be achieved, independently of isospin assumptions, via e.g. counting single versus double tagged semileptonic $B$ decays [71], from which $r_{+0}$ may be extracted under the assumption $f_{00}+f_{+-}=1$ and uncorrelated reconstruction efficiencies, or via e.g. measuring relative branching ratios for inclusive semileptonic processes [72, 14], in which isospin violation is expected to be suppressed to order $1 / \mathrm{m}_{b}^{2}$. Combination of available data for these two approaches yields $r_{+0}=1.027 \pm 0.037$ [70].

\section{Conclusion}

The last two decades have seen enormous progress in the understanding of $B$ meson mixing and mixing-related $C P$ violation, both in terms of precise experimental measurements of the underlying constants of nature and in terms of the theoretical understanding of their values within the Standard Model. We now have precise measurements or stringent limits on the mass splitting, width splitting, and mixing phase in both the $B^{0}$ and $B_{s}^{0}$ systems, while mixing-induced $C P$ violation is being precisely measured or constrained in an ever increasing number of final states. With the LHCb upgrade [73] and Belle II [74] detectors due to come online in the next few years, and none of the fundamental measurements yet systematically limited, we can expect this progress to continue. Important contributions, particularly as regards $\phi_{s}$ and $\Delta \Gamma_{d}$ can also be expected from CMS and ATLAS, and in particular it is realistic to expect the $B_{s}^{0}$ mixing phase to be measured significantly away from zero even at the Standard Model value within the next decade. Recently the LHCb collaboration has proposed a Phase II upgrade of its detector [75], to take data in the HL-LHC period, which would collect $300 \mathrm{fb}^{-1}$, and enable not only a single-experiment observation of $\phi_{s}$ in multiple independent decay modes, but also make it possible to see evidence for a non-zero $\Delta \Gamma_{d}$ at its Standard Model value.

Computation and further control of subleading corrections in the extraction of CKM angles and matrix elements from $B$ mixing has proceeded apace with new state-of-the-art lattice calculations. The former, particularly extraction of $\sin 2 \beta$ from $b \rightarrow c \bar{c} s$ decays, has been the subject of various recent studies, with the emerging conclusion that theory uncertainties are likely smaller than the attainable experimental precision. Recent direct calculations as well as new flavor symmetry analyses will provide complementary handles to decide this question. State-of-the-art lattice results 
for $B$-mixing matrix elements indicate a possible evolving tension between predicted and measured mass splittings $\Delta m_{d, s}$, or in the context of the $b \rightarrow d$ CKM unitarity triangle, a tension between $\Delta m_{s, d}$ and $\varepsilon_{K}$ allowed regions. Apart from constrained minimal violation theories, which seem unable to account for this tension, at present potential theoretical implications are relatively unexplored.

\section{References}

[1] ARGUS collaboration, H. Albrecht et al., Observation of BO - anti-BO Mixing, Phys. Lett. B192 (1987) 245-252.

[2] B. Hamilton, "Summary of WG2", in proceedings of "9th International Workshop on the CKM Unitarity Triangle", vol. PoS(CKM2016)015, 2017.

[3] LHCb COllaboration collaboration, R. Aaij et al., Resonances and $C P$-violation in $B_{s}^{0}$ and $\bar{B}_{s}^{0} \rightarrow J / \psi K^{+} K^{-}$decays in the mass region above the $\phi$, LHCb-PAPER-2017-008.

[4] M. Artuso, G. Borissov and A. Lenz, CP violation in the $B_{s}^{0}$ system, Rev. Mod. Phys. 88 (2016) 045002, [1511.09466].

[5] P. Reznicek, "Latest ATLAS results on $\phi_{s}$ ", in proceedings of "9th International Workshop on the CKM Unitarity Triangle", vol. PoS(CKM2016)083, 2017.

[6] ATLAS collaboration, G. Aad et al., Measurement of the CP-violating phase $\phi_{s}$ and the $B_{s}^{0}$ meson decay width difference with $B_{s}^{0} \rightarrow J / \psi \phi$ decays in ATLAS, JHEP 08 (2016) 147, [1601.03297].

[7] CMS collaboration, V. Khachatryan et al., Measurement of the CP-violating weak phase $\phi_{s}$ and the decay width difference $\Delta \Gamma_{s}$ using the $B_{s}^{0} \rightarrow J / \psi \phi(1020)$ decay channel in pp collisions at $\sqrt{s}=8 \mathrm{TeV}$, Phys. Lett. B757 (2016) 97-120, [1507.07527].

[8] LHCB COLlaboration collaboration, G. Cowan, Measurements of $\phi_{s}$ at LHCb, .

[9] U. Nierste, "Penguin pollution in $\beta$ and $\beta_{s}$ ", in proceedings of "9th International Workshop on the CKM Unitarity Triangle", vol. PoS(CKM2016)081, 2017.

[10] LHCB COLLABORATION collaboration, R. Aaij et al., Measurement of CP violation parameters and polarisation fractions in $B_{s}^{0} \rightarrow J / \psi \bar{K}^{* 0}$ decays, JHEP 11 (2015) 082 LHCb-PAPER-2015-034, CERN-PH-EP-2015-224, [1509.00400].

[11] LHCb COllaboration collaboration, R. Aaij et al., Measurement of the CP-violating phase $\beta$ in $\bar{B}^{0} \rightarrow J / \psi \pi^{+} \pi^{-}$decays and limits on penguin effects, Phys. Lett. B742 (2015) 38 CERN-PH-EP-2014-268, LHCb-PAPER-2014-058, [1411.1634].

[12] LHCb COllaboration collaboration, R. Aaij et al., Measurement of $C P$ violation in $B_{s}^{0} \rightarrow \phi \phi$ decays, Phys. Rev. D90 (2014) 052011 CERN-PH-EP-2014-150, LHCb-PAPER-2014-026, [14 07.2222 ].

[13] LHCB collaboration, T. L. Collaboration, Measurement of time-dependent CP-violating asymmetries in $B^{0} \rightarrow \pi^{+} \pi^{-}$and $B_{s}^{0} \rightarrow K^{+} K^{-}$decays at $L H C b$,

[14] Heavy Flavor Averaging Group collaboration, Y. Amhis et al., Averages of b-hadron, c-hadron, and $\tau$-lepton properties as of summer 2016, 1612.07233.

[15] R. Fleischer, New strategies to extract Beta and gamma from $B(d) \rightarrow p i+$ pi- and $B(S) \longrightarrow K+K$-, Phys. Lett. B459 (1999) 306-320, [hep-ph/ 9903456 ].

[16] LHCb COllaboration collaboration, R. Aaij et al., Determination of $\gamma$ and $-2 \beta_{s}$ from charmless two-body decays of beauty mesons, Phys. Lett. B739 (2015) 1 CERN-PH-EP-2014-207, LHCb-PAPER-2014-045, [1408 . 4368]. 
[17] BABAR collaboration, J. P. Lees et al., Measurement of CP Asymmetries and Branching Fractions in Charmless Two-Body B-Meson Decays to Pions and Kaons, Phys. Rev. D87 (2013) 052009, [1206.3525].

[18] BELLE collaboration, I. Adachi et al., Measurement of the CP violation parameters in $B^{0} \rightarrow \pi^{+} \pi^{-}$ decays, Phys. Rev. D88 (2013) 092003, [1302.0551].

[19] LHCB COllaboration collaboration, R. Aaij et al., A precise measurement of the $B^{0}$ meson oscillation frequency, Eur. Phys. J. C76 (2016) 412 LHCb-PAPER-2015-031, CERN-EP-2016-084, [1604.03475].

[20] LHCb Collaboration collaboration, R. Aaij et al., Precision measurement of the $B_{s}^{0}-\bar{B}_{s}^{0}$ oscillation frequency in the decay $B_{s}^{0} \rightarrow D_{s}^{-} \pi^{+}$, New J. Phys. 15 (2013) 053021 CERN-PH-EP-2013-054, LHCb-PAPER-2013-006, [1304 . 4741].

[21] F. Palla, Cms : What the 40 mhz track trigger bring us?, http: / / inyurl.com/ Is jwcfx.

[22] A. Lenz and U. Nierste, Numerical Updates of Lifetimes and Mixing Parameters of B Mesons, in CKM unitarity triangle. Proceedings, 6th International Workshop, CKM 2010, Warwick, UK, September 6-10, 2010, 2011. 1102.4274.

[23] T. Gershon, $\Delta \Gamma_{d}:$ A Forgotten Null Test of the Standard Model, J. Phys. G38 (2011) 015007 , [1007.5135].

[24] G. Borissov and B. Hoeneisen, Understanding the like-sign dimuon charge asymmetry in p $\bar{p}$ collisions, Phys. Rev. D87 (2013) 074020, [1303.0175].

[25] LHCB COllaboration collaboration, R. Aaij et al., Measurements of the $B^{+}, B^{0}, B_{s}^{0}$ meson and $\Lambda_{b}^{0}$ baryon lifetimes, JHEP 04 (2014) 114 LHCb-PAPER-2013-065, CERN-PH-EP-2014-018, [1402.2554].

[26] ATLAS collaboration, M. Aaboud et al., Measurement of the relative width difference of the $B^{0}-\bar{B}^{0}$ system with the ATLAS detector, JHEP 06 (2016) 081, [1605. 07485 ].

[27] BABAR collaboration, J. P. Lees et al., Tests of CPT symmetry in $B^{0}-\bar{B}^{0}$ mixing and in $B^{0} \rightarrow c \bar{c} K^{0}$ decays, Phys. Rev. D94 (2016) 011101, [1605.04545].

[28] LHCB COLlaboration collaboration, R. Aaij et al., First measurement of the photon polarization in radiative $B_{s}^{0}$ decays, 1609.02032 LHCb-PAPER-2016-034, CERN-EP-2016-210, [1609. 02032 ].

[29] F. Muheim, Y. Xie and R. Zwicky, Exploiting the width difference in $B_{s} \rightarrow \phi \gamma$, Phys. Lett. B664 (2008) 174-179, [0802.0876].

[30] BELLE collaboration, P. Vanhoefer et al., Study of $\mathbf{B}^{\mathbf{0}} \rightarrow \rho^{+} \rho^{-}$decays and implications for the CKM angle $\phi_{2}$, Phys. Rev. D93 (2016) 032010, [1510.01245].

[31] BABAR collaboration, B. Aubert et al., Measurement of Time-Dependent CP Asymmetry in BO $\rightarrow c$ anti-c K(*)O Decays, Phys. Rev. D79 (2009) 072009, [0 902.1708 ].

[32] I. Adachi et al., Precise measurement of the CP violation parameter sin2phil in BO -> (c-bar c)KO decays, Phys. Rev. Lett. 108 (2012) 171802, [1201.4643].

[33] LHCB COllaboration collaboration, R. Aaij et al., Measurement of the time-dependent CP asymmetry in $B^{0} \rightarrow J / \psi K_{\mathrm{S}}^{0}$ decays, Phys. Lett. B721 (2013) 24 LHCb-PAPER-2012-035, CERN-PH-EP-2012-331, [1211.6093].

[34] LHCB COllaboration collaboration, R. Aaij et al., Measurement of the time-dependent $C P$ asymmetries in $B_{S}^{0} \rightarrow J / \psi K_{\mathrm{S}}^{0}$, JHEP 06 (2015) 131 CERN-PH-EP-2015-064, LHCb-PAPER-2015-005, [1503.07055]. 
[35] Belle, BABAR collaboration, A. Abdesselam et al., First Observation of CP Violation in $\bar{B}^{0} \rightarrow D_{\mathrm{CP}}^{(*)} h^{0}$ Decays by a Combined Time-Dependent Analysis of BABAR and Belle Data, Phys. Rev. Lett. 115 (2015) 121604, [1505.04147].

[36] M. John, "Summary of WG5", in proceedings of "9th International Workshop on the CKM Unitarity Triangle", vol. PoS(CKM2016)018, 2017.

[37] J. Brod and J. Zupan, The ultimate theoretical error on $\gamma$ from B $\rightarrow$ DK decays, JHEP 01 (2014) 051, [1308.5663].

[38] LHCb COllaboration collaboration, A. Dziurda, Measurement of $\gamma$ from a time-dependent analysis of $B_{s}^{0} \rightarrow D_{s}^{ \pm} K^{\mp}$, .

[39] LHCB COLlaboration collaboration, R. Aaij et al., Measurement of the CKM angle $\gamma$ from a combination of LHCb results, JHEP 12 (2016) 087 LHCb-PAPER-2016-032, CERN-EP-2016-270, [1611.03076].

[40] LHCB COLlaboration collaboration, R. Aaij et al., Measurement of CP observables in $B^{ \pm} \rightarrow D K^{ \pm}$ and $B^{ \pm} \rightarrow D \pi^{ \pm}$with two- and four-body D meson decays, Phys. Lett. $\mathbf{B 7 6 0}$ (2016) 117 LHCb-PAPER-2016-003, CERN-EP-2016-065, [1603. 08993].

[41] LHCB COLlabORATION collaboration, A. Birnkraut, Measurement of $\gamma$ from a time-dependent analysis of $B \rightarrow D \pi$, .

[42] BABAR collaboration, B. Aubert et al., Measurement of time-dependent CP-violating asymmetries and constraints on $\sin (2 \beta+\gamma)$ with partial reconstruction of $B \rightarrow D^{* \mp} \pi^{ \pm}$decays, Phys. Rev. D71 (2005) 112003, [hep-ex/0504035].

[43] BABAR collaboration, B. Aubert et al., Measurement of time-dependent CP asymmetries in $B^{0} \rightarrow D^{(*)}$ $+-\pi^{\mp}$ and $B^{0} \rightarrow D^{ \pm} \rho^{\mp}$ decays, Phys. Rev. D73 (2006) 111101, [hep-ex/ 0602049 ].

[44] BELLE collaboration, S. Bahinipati et al., Measurements of time-dependent CP asymmetries in $B \rightarrow D^{* \mp} \pi^{ \pm}$decays using a partial reconstruction technique, Phys. Rev. D84 (2011) 021101 , [1102.0888].

[45] BELLE collaboration, F. J. Ronga et al., Measurements of $\mathrm{CP}$ violation in $\mathrm{BO} \longrightarrow \mathrm{D}^{*_{-}}$pi+ and $\mathrm{BO} \longrightarrow$ D- pi+ decays, Phys. Rev. D73 (2006) 092003, [hep-ex/ 0604013 ].

[46] Fermilab LATtice, MILC collaboration, A. Bazavov et al., $B_{(s)}^{0}$-mixing matrix elements from lattice QCD for the Standard Model and beyond, Phys. Rev. D93 (2016) 113016, [1602 . 03560].

[47] Y. Aoki, T. Ishikawa, T. Izubuchi, C. Lehner and A. Soni, Neutral B meson mixings and B meson decay constants with static heavy and domain-wall light quarks, Phys. Rev. D91 (2015) 114505, [1406.6192].

[48] ETM collaboration, N. Carrasco et al., B-physics from $N_{f}=2$ tmQCD: the Standard Model and beyond, JHEP 03 (2014) 016, [1308 . 1851$].$

[49] A. Bazavov et al., Neutral B-meson mixing from three-flavor lattice QCD: Determination of the SU(3)-breaking ratio $\xi$, Phys. Rev. D86 (2012) 034503, [1205. 7013 ].

[50] C. Albertus et al., Neutral B-meson mixing from unquenched lattice QCD with domain-wall light quarks and static b-quarks, Phys. Rev. D82 (2010) 014505, [1001.2023].

[51] HPQCD collaboration, E. Gamiz, C. T. H. Davies, G. P. Lepage, J. Shigemitsu and M. Wingate, Neutral B Meson Mixing in Unquenched Lattice QCD, Phys. Rev. D80 (2009) 014503, [0 902.1815 ]. 
[52] E. Dalgic, A. Gray, E. Gamiz, C. T. H. Davies, G. P. Lepage, J. Shigemitsu et al., $B_{s}^{0}-\bar{B}_{s}^{0}$ mixing parameters from unquenched lattice QCD, Phys. Rev. D76 (2007) 011501, [hep-lat/ 0610104 ].

[53] CKMfitter Group collaboration, J. Charles et al., CP violation and the CKM matrix: Assessing the impact of the asymmetric B factories, Eur. Phys. J. C41 (2005) 1-131, [hep-ph/ 0406184 ].

[54] J. Charles et al., Current status of the Standard Model CKM fit and constraints on $\Delta F=2$ New Physics, Phys. Rev. D91 (2015) 073007, [1501.05013].

[55] A. J. Buras, P. Gambino, M. Gorbahn, S. Jager and L. Silvestrini, Universal unitarity triangle and physics beyond the standard model, Phys. Lett. B500 (2001) 161-167, [hep-ph/ 0007085 ].

[56] A. J. Buras, Minimal flavor violation, Acta Phys. Polon. B34 (2003) 5615-5668, [hep-ph/0310208].

[57] M. Blanke, A. J. Buras, D. Guadagnoli and C. Tarantino, Minimal Flavour Violation Waiting for Precise Measurements of $\Delta M_{s}, S_{\psi \phi}, A_{S L}^{s},\left|V_{u b}\right|, \gamma$ and $B_{s, d}^{0} \rightarrow \mu^{+} \mu^{-}, J H E P 10$ (2006) 003, [hep-ph/0604057].

[58] M. Blanke and A. J. Buras, Lower bounds on $\Delta M\left(s, d^{\prime}\right.$ from constrained minimal flavour violation, JHEP 05 (2007) 061, [hep-ph/ 0610037 ].

[59] M. Blanke and A. J. Buras, Universal Unitarity Triangle 2016 and the tension between $\Delta M_{s, d}$ and $\varepsilon_{K}$ in CMFV models, Eur. Phys. J. C76 (2016) 197, [1602.04020].

[60] M. Blanke, CMFV models facing the recent progress in lattice calculations of $B_{s, d}$ mixing, PoS CKM2016 (2016) 089, [1612.08943].

[61] H. Boos, T. Mannel and J. Reuter, The Gold plated mode revisited: $\operatorname{Sin}(2$ beta) and BO $\rightarrow J / P s i K(S)$ in the standard model, Phys. Rev. D70 (2004) 036006, [hep-ph/ 0403085 ].

[62] M. Ciuchini, M. Pierini and L. Silvestrini, The Effect of penguins in the $B(d) \rightarrow J / \psi K^{0}$ CP asymmetry, Phys.Rev.Lett. 95 (2005) 221804, [hep-ph/ 0507290$].$

[63] H.-n. Li and S. Mishima, Penguin pollution in the BO $\rightarrow$ J/psi K(S) decay, JHEP 03 (2007) 009, [hep-ph/0610120].

[64] S. Faller, M. Jung, R. Fleischer and T. Mannel, The Golden Modes $B 0 \rightarrow J / \psi K_{S, L}$ in the Era of Precision Flavour Physics, Phys.Rev. D79 (2009) 014030, [0 809.0842$].$

[65] M. Ciuchini, M. Pierini and L. Silvestrini, Theoretical uncertainty in sin 2 $\beta$ : An Update, 1102.0392.

[66] M. Jung, Determining weak phases from B $\rightarrow J / \psi P$ decays, Phys.Rev. D86 (2012) 053008, [1206.2050].

[67] K. De Bruyn and R. Fleischer, A Roadmap to Control Penguin Effects in $B_{d}^{0} \rightarrow J / \psi K_{\mathrm{S}}^{0}$ and $B_{s}^{0} \rightarrow J / \psi \phi, J H E P 1503$ (2015) 145, [1412.6834].

[68] P. Frings, U. Nierste and M. Wiebusch, Penguin contributions to CP phases in $B_{d, s}$ decays to charmonium, Phys. Rev. Lett. 115 (2015) 061802, [1503.00859].

[69] Z. Ligeti and D. J. Robinson, Towards more precise determinations of the quark mixing phase $\beta$, Phys. Rev. Lett. 115 (2015) 251801, [1507.06671].

[70] M. Jung, Branching ratio measurements and isospin violation in B-meson decays, Phys. Lett. B753 (2016) 187-190, [1510.03423].

[71] BABAR collaboration, B. Aubert et al., Measurement of the branching fraction of $\Upsilon(4 S) \rightarrow B^{0} \bar{B}^{0}$, Phys. Rev. Lett. 95 (2005) 042001, [hep-ex/ 0504001 ]. 
[72] BELLE collaboration, N. C. Hastings et al., Studies of BO - anti-BO mixing properties with inclusive dilepton events, Phys. Rev. D67 (2003) 052004, [hep-ex/ 0212033 ].

[73] LHCB COLLABORATION collaboration, I. Bediaga et al., Framework TDR for the LHCb Upgrade: Technical Design Report, Tech. Rep. CERN-LHCC-2012-007. LHCb-TDR-12, Apr, 2012.

[74] BeLle-II collaboration, T. Abe et al., Belle II Technical Design Report, 1011.0352.

[75] LHCB COLlaboration collaboration, R. Aaij et al., Expression of Interest for a Phase-II LHCb Upgrade: Opportunities in flavour physics, and beyond, in the HL-LHC era, Tech. Rep. CERN-LHCC-2017-003, CERN, Geneva, Feb, 2017. 